\title{
Análisis de Presión y Derivada de Presión sin Curvas Tipo para un Pozo Horizontal en un Yacimiento de dos Capas
}

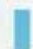

Kittiphang Jangkittinarukorn. PTT Exploration and Production Public Company. Limited 555 Vibhavadi-Rangsit Rd. Chatuchak, Bangkok I0900 Thailand.

Djebbar Tiab. School of Petroleum and Geological Engineering. The University of Oklahoma. T30I Sarkeys Energy Center. IOOE. Boyd St.. Norman. Ok 73019. USA.

Freddy Humberto Escobar. Ing. de Petróleos. Ph.D. Profesor Titular. USCD.

\section{Resumen}

L.te artículo presenta tres aplicaciones diferentes para interpretar pruebas de presión en pozos horizontales eliminando el uso de las curvas tipo excluyendo efectos de almacenamiento y daño. Esta metodología, primero intraducida por Tiab' en 1993, fue extendida Jongkittinarukorn² para proporcionar una herramienta de pruebas de presión para (a) pozo horizontal en un yacimiento de dos capas con flujo cruzado, (b) pozo harizontal interceptando un yacimienta de dos capas. у с) pazo horizontal ondulando en un yacimiento de dos capas.

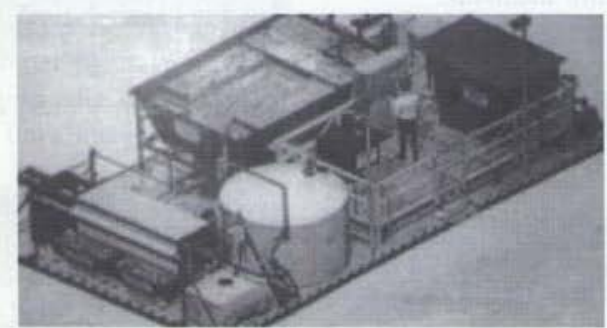


Análisis de presiôn y derivada de presiön sin curvas tipo para un pozo borizontal en un yacimiento de dos capas

\section{Introducción}

La interpretación de prucbas de presión en pozos horizontales es más difícil de realizar que en pozos verticales debido a la geometría del pozo, a la heterogeneidad del medio y a la variedad de regímenes de flujo. Kuchuk and Habashy ${ }^{3}$ resolvieron la ecuación de difusividad para sistemas multicapas (sD) para estudiar el comportamiento de la presión en pozos horizontales. Para mayor precisión y evitar efectos de orientación de la malla, Jongkittinarukorn usó el método de elementos de frontera, MEF, para diferentes condiciones de frontera y configuraciones de pozo. Además, extendió la Tiab's Direct Synthesis Technique a ocho casos diferentes, tres de los cuales se presentan en este artículo. Se presentan ejemplos típicos y procedimientos paso a paso para validar su aplicación. Otra aplicaciones del MEF a pozos horizontales fueron hechas por Koh y Tiab ${ }^{+} \mathrm{y}$ Jongkittinarukorn ${ }^{5}$ entre otras. . +

\section{Casa I: Paza Harizontal en un Yacimiento Bi- Capas con Fluja-Eruzado}

El modelo de yacimiento considera el pozo centrado en la capa superior. Las capas poseen espesor igual y constante. La presión y su derivada presentan varios puntos característicos y porciones rectas:

1) Durante el primer flujo radial, la caída de presión y su derivada en unidades de campos se expresan respectivamente mediante:

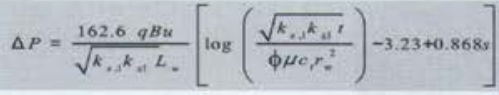

$$
t^{*} \Delta P^{\prime}=\frac{70.6 q B \mu}{\sqrt{k_{x, 1} k_{z, 1} L_{w}}}
$$

La derivada puede emplearse para estimar la permeabilidad radial $\left(k_{x, 1} k_{z, 1}\right)^{0.5}$ de la Ec. (1.2). De la Ec. 1.1, gráfico semilog, se obtiene una recta de cuya pendiente se tiene:

$$
\sqrt{k_{x, 1} k_{z, 1}} \frac{162.6 q B \mu}{m_{1} L_{w}}
$$

El daño mecánico se calcula de:

$$
s=1.151\left[\left(\frac{P_{1}-P_{1, k}}{m_{1}}\right)-\log \left(\frac{\sqrt{k_{x, 1} k_{z, 1}}}{\phi \mu c_{,} r_{w}^{2}}\right)+3.23\right]
$$

2) Durante el segundo flujo radial, la relación entre la derivada y la permeabilidad es:

$t_{D} P_{D}^{\prime}=-0.0327 x^{3}+0.204 x^{4}-0.387 x^{3}+0.011 x^{2}+0.711 x+0.5=\frac{k_{x, 1} L_{-}\left(t^{*} \Delta P^{\prime}\right)}{141.2 q B_{\mu}}$

donde $x=\log \left(k_{x, 1} / k_{x, 2}\right)$. Una vez se conoce la permeabilidad en la dirección $\mathrm{x}$ en la capa superior, la Ec. 1.5 se usa para hallar $k_{x, 2}$

s) Durante el periodo pseudoestable, la presión adimensional y la derivada están dadas por:

$P_{D} \frac{L_{y}}{L_{w}} \sqrt{\frac{k_{z, 1}}{k_{x, 1}}}=2 \pi t_{D A}+\frac{1}{2} \ln \left(\frac{2.246 A}{C_{A} r_{w h}^{2}}\right)+\frac{L_{y}}{L_{w}} s+s_{p t}$

Dande:

$$
t_{D} * P_{D}^{\prime}=\frac{2 \pi r_{w}^{2}}{A} \frac{L_{w}}{L_{y}} t_{D}
$$

$t_{D A t}=\frac{0.0002637 \sqrt{k_{s, 1} k_{z-1}} t}{\phi \mu c_{i} A}$

$$
A=L x L z
$$

$r_{w h}=\frac{1}{2}\left[\sqrt[4]{\frac{k_{x}}{k_{x}}}+\sqrt[4]{\frac{k_{x}}{k_{x}}}\right] r_{w}$

Las Ecs. (1.8) y (1.9) expresadas en unidades de campo:;

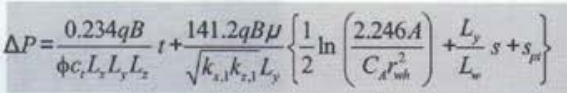

$$
t^{*} \Delta P^{\prime}=\frac{0.234 q B}{\phi c_{1} L_{x} L_{y} L_{z}} t
$$

Un gráfico cartesiano de $\Delta \mathrm{P}$ vs. tiempo da una línea recta de pendiente que corresponde al primer término de la derecha de la Ec. 1.11 e intercepto que corresponde al segundo término de la misma. 
expresión. De esta pendiente se puede determinar el volumen del yacimiento $\left(L_{x} L_{y} L_{z}\right)$.

4) La caída de presión promedia durante flujo pseudoestable es:

$$
\left(\bar{P}-P_{w f f}\right)=\frac{141.2 q B \mu}{\sqrt{k_{x, 1} k_{z, 1}} L_{y}}\left\{\frac{1}{2} \ln \left(\frac{2.246 A}{C_{A} r_{w+h}^{2}}\right)+\frac{L_{y}}{L_{w}} s+s_{p=}\right\}
$$

De donde es fácil despejar la presión promedia del yacimiento se tiene:

5) El índice de productividad, unidades de campo, se calcula de:

$$
J=\frac{q}{\left(\bar{P}-P_{w j}\right)}=\frac{0.00708 \sqrt{k_{x, 1} k_{z, 1}} L_{y}}{B \mu\left\{\frac{1}{2} \ln \left(\frac{2.246 A}{C_{A} r_{m h}^{2}}\right)+\frac{L_{y}}{L_{w}} s+s_{p u}\right\}}
$$

6) La permeabilidad direccional puede obtenerse de la desviación de la derivada. El radio de investigación está dado por:

$r=\sqrt{\frac{k t}{948 \phi \mu c}}$

El proceso paso a paso para calcular los parámetros del yacimiento es:

Paso l: Construya gráfico de derivada, semilog y cartesiano. Use la Ec. (1.2) para hallar $\left(k_{x, 1} k_{z, 1}\right)^{1 / 2}$ También, puede usar la Ec. (1.3) usando el gráfico semilog.

Paso 2: Identifique el estado pseudoestable y halle la pendiente en el gráfico cartesiano. Use la Ec. (1.11) para hallar el volumen bruto del yacimiento.

Paso 3: Calcule la presión promedia usando la Ec. (1.13) y el intercepto de la línea mencionada en el paso 2.

Paso 4: Calcule el índice de productividad con la Ec. (1.14).

Paso 5: Utilice la derivada para hallar la desviación de la línea de tendencia. Use la Ec. (1.15) para hallar permeabilidad.

Paso 6: Determine $k_{x, 1}$ con la permeabilidad del paso 1 y la otra permeabilidad del paso 5 .

Paso 7: Identifique el Segundo flujo radial. Resuelva la Ec. Ec. (1.5) para hallar $\left(k_{x, 1} / k_{x, 2}\right)$. Calcule $k_{x, 2}$ mediante $k_{x, 2}=k_{x, 1} / R_{k}$.
Ejempla. Un pozo horizontal se halla $5 \mathrm{ft}$ por debajo del tope y a $5 \mathrm{ft}$ de la interfase. Los datos de presión se dan en las Figs. 1.1, y 1.2. Para esta prueba sintética otra información relevante es:
$L_{\mathrm{w}}=100 \mathrm{ft}$.
$q=50 \mathrm{stb} / \mathrm{d}$
$B=1.2 \mathrm{rb} / \mathrm{stb}$
$\mu=1.0 \mathrm{cp}$
$\phi=20 \%$
$r_{w}=0.5 \mathrm{t}$

Solución: Durante el primer flujo radial la derivada es 1.34 psi, Fig. 1.1, usando la Ec. 1.2 se tiene $\left(k_{x, 1} k_{x, 1}\right)^{1 / 2}=31.61 \mathrm{md}$. Del gráfico semilog, Fig. 1.2, la pendiente es $3 \mathrm{psi} /$ ciclo. De la Ec. 1.3 se tiene $\left(k_{x, 1} k_{z, 1}\right)^{1 / 2}=32.52 \mathrm{md}$. Durante estado pseudoestable la pendiente e intercepto son $0.05 \mathrm{psi} /$ ciclo y 14.1 psi. De modo que usando la pendiente de la Ec. 1.11 se obtiene $L_{x} L_{y} L_{x}=0.94 \times 10^{8} \mathrm{ft}^{3}$. Del intercepto resulta una presión promedia $=P_{+}$ 14.1 psi. Usando la Ec. 1.15, J = $3.55 \mathrm{stb} / \mathrm{d} / \mathrm{psi}$. De la Fig. 1.3, la presión se desvía a las 0.007 hr. De la Ec. 1.15 y teniendo $\mathrm{r}=5 \mathrm{ft}$ se halla $k_{z, 1}$ de $10.16 \mathrm{md}$. También, $k_{x, 1}=98.4 \mathrm{md}$. Con el Segundo flujo radial y la Ec. 1.5 se halla $k_{x, 2}=10.07 \mathrm{md}$.

\section{Caso 2: Pozo Harizontal Interceptando un Yacimienta Bi-Capas}

El modelo de yacimiento no presenta flujo cruzado. $\mathrm{El}$ pozo se inclina un ángulo q con respecto al plano horizontal y penetra igualmente ambas capas. Las "huellas digitales" son ahora:

1) Durante el primer flujo radial, la derivada puede expresarse como:

$t_{D}^{*} P_{D}^{\prime}=c_{1} R_{k}^{5}-c_{2} R_{k}^{4}+c_{3} R_{k}^{3}-c_{4} R_{k}^{2}+c_{5} R_{k}+c_{6}=\frac{k_{x, t} L_{w}(t * \Delta P)}{141.2 q B \mu}$ donde, para $\mathrm{Q}^{\circ} \mathrm{fq} \mathrm{f} \mathrm{qQ}^{\square}$.

$c_{1}=7.234 * 10^{-12} \theta^{2}-1.232 * 10^{-9} \theta+7.740 * 10^{-8}$ (2.2a)

$c_{2}=2.140 * 10^{-9} \theta^{2}-2.496 * 10^{7} \theta+1.237 * 10^{-6}$

$c_{3}=8.044^{*} 10^{-8} \theta^{2}-1.316^{*} 10^{-5} \theta+7.646^{*} 10^{-4}$

$c_{4}=-2.596 * 10^{-6} \theta^{2}+4.137 * 10^{-4} \theta-2.284 * 10^{2}$

$c_{5}=4.091 * 10^{-5} \theta^{2}-6.339 * 10^{-3} \theta+3.312 * 10^{-1}$

$c_{6}=1.734 * 10^{-4} \theta^{2}-2.498^{*} 10^{-2} \theta+1.258^{*} 10^{-0}$

q) Durante el segundo flujo radial, al derivada se expresa por:

$t_{D}{ }^{*} P_{D}{ }^{\prime}=c_{1} R_{k}^{5}-c_{2} R_{k}^{4}+c_{3} R_{k}^{3}-c_{4} R_{k}^{2}+c_{5} R_{k}+c_{6}$ 
Anälisis de presión y derizada de presión sin curvas tipo para un pozo borizontal еи ии yacimiento de dos capas

donde, para $0^{\circ} \leq \theta \leq 90^{\circ}$,

$c_{1}=1.829 * 10^{-12} \theta^{2}-2.692 * 10^{-10} \theta+3.191 * 10^{-8}$

$c_{2}=3.008 * 10^{-10} \theta^{2}+4.303^{*} 10^{8} \theta-4.981 * 10^{-6}$

$c_{3}=1.944 * 10^{\circ} \mathrm{\theta}-2.689^{*} 10^{\circ} \theta+3.001 * 10^{-4}$

$c_{4}=-5.910^{*} 10^{-7} \theta^{2}+7.956 * 10^{-5} \theta-8.695 * 10^{-3}$

$c_{5}=8.385^{*} 10^{-6} \theta^{2}-1.113^{*} 10^{-3} \theta+1.224 * 10^{-1}$

$c_{6}=4.262 * 10^{-6} \theta^{2}-4.687^{*} 10^{-4} \theta+4.236^{*} 10^{-1}$

(2.4a)

(2.4b)

(2.4c)

(2.4d)

(2.4e)

3) Durante la transición entre el periodo pseudoestable existe una recta de pendiente 0.8 cuya longitud ( $L_{p \rho s s}$ ) es función de $R_{k}$. Esta relación que sirve para hallar $R_{k}$ está dada por:

\& $\quad(2.5)$ $L_{h \mathrm{~m}}=1.451 \times 10^{-4} R_{x}^{3}-3.992 \times 10^{-7} R_{x}^{4}+4.054 \times 10^{5} R_{x}^{2}-0.00190 R_{x}^{2}+0.0631 R_{x}^{2}-0.0612$

4) Durante el estado pseudoestable la presión adimensional está dada por:

$P_{D} \sqrt{\frac{k_{y}}{k_{x}}}=2 \pi t_{D A}+\frac{1}{2} \ln \left(\frac{2.246 A}{C_{A} r_{w}^{2}}\right)+s^{*}$,

donde: $\quad t_{D A}=\frac{0.0002637 \sqrt{k_{x, 1} k_{y, 1}}}{\phi \mu c_{t} A} t$

$A=L_{x} L_{y}$

La Ec. (2.6) en unidades de campo es:

$$
\Delta P=\frac{0.234 q B}{\phi c_{z} L_{x} L_{y} L_{z}} t+\frac{141.2 q B \mu}{\sqrt{k_{x, 1} k_{y, 1} L_{z}}}\left\{\frac{1}{2} \ln \left(\frac{2.246 A}{C_{A} r_{w}^{2}}\right) s\right\}
$$

Un gráfico cartesiano de DP versus tiempo da una línea recta cuya pendiente (para hallar volumen, $\left.L_{x} L_{y} L_{z}\right)$ y corte son:

$$
m_{p a t}=\frac{0.234 q B}{\phi c_{r} L_{x} L_{y} L_{z}}
$$

$$
I_{p u s}=\frac{141.2 q B \mu}{\sqrt{k_{x, 1} k_{y, 1} L_{z}}}\left\{\frac{1}{2} \ln \left(\frac{2.246 A}{C_{d} r_{w}^{2}}\right)+s\right\}
$$

5) La presión promedia durante estado pseudoestable está dada por:

$$
\left(\bar{P}-P_{v-}\right)=I_{p \mu}=\frac{141.2 q B \mu}{\sqrt{k_{x, j} k_{y, 1} L_{z}}}\left\{\frac{1}{2} \ln \left(\frac{2.246 A}{C_{A} r_{z}^{2}}\right)+s\right\}
$$

6) El índice de productividad es:

$$
J=\frac{q}{\left(\bar{P}-P_{w f}\right)}=\frac{0.00708 \sqrt{k_{x, 1} k_{y, d}} L_{z}}{B \mu\left\{\frac{1}{2} \ln \left(\frac{2.246 A}{C_{A} r_{v}^{2}}\right)+s\right\}}
$$

7) La desviación de la derivada puede usarse para estimar la permeabilidad direccional o la distancia del pozo a la frontera o interfase. El radio de investigación se expresa por la Ec. 1.15.

El procedimiento paso a paso para estimar los parámetros del yacimiento es:

Paso 1: Construya los mismos gráficos del caso 1. Identifique el periodo de transición antes del estado pseudoestable. Calcule la longitud de la porción recta $\left(L_{\text {ppss }}\right)$ durante este periodo. Use la Ec. (2.5) para determinar $R_{k}$.

Paso 2: Identifique el primer flujo radial. Con la Ec. (2.1) halle $k_{x, 1}$

Paso 3: Halle $k_{x, 2}$ por medio de $k_{x, 2}=k_{x, 1} / R_{k}$

Paso 4: Del gráfico cartesiano determine la pendiente $\left(m_{\text {pss }}\right)$ y el corte $\left(I_{\text {pss }}\right)$.

Paso 5: De la pendiente halle el volumen del yacimiento usando la Ec. 2.10.

Paso 6: Halle la presión promedia con la Ec. (2.13). Paso 7: Calcule $J$ con la Ec. 2.13 .

Paso 8: De la derivada, identifique el tiempo de su desviación y use la Ec. 1.15 para hallar la distancia del pozo a la frontera o a la interfase.

\section{Ejemplo}

Esta prueba simulada es para un pozo en el centro de un yacimiento. Los datos de presión se dan en las Figs. 2.1 y 2.2. otros datos son:

$L_{w}=100 \mathrm{ft}$.
$\mu=1 \mathrm{cp}$
$s=0$
$k_{x, 2}=0.25 \mathrm{md}$
$k_{z, 1}=1 \mathrm{md}$

$q=40 \mathrm{stb} / \mathrm{d}$

$\phi=0.1$

$r_{\mathrm{w}}=0.5 \mathrm{ft}$

$k_{y, 1}=10 \mathrm{md}$

$k_{z, 2}=0.025 \mathrm{md}$

$B=1.1 \mathrm{rb} / \mathrm{stb}$

$\phi_{2}=0.1$ $c_{t}=0.00001 \mathrm{psi}^{-1}$

$k_{x, 1}=10 \mathrm{md}$

$k_{y, 2}=0.25 \mathrm{md}$

$\phi_{1}=0.1$ 
Solución: Durante la transición antes del estado pseudoestable existe una recta con pendiente de 0.8 . Su longitud $L_{p p s s}=0.59$. De la Ec. $2.5, R_{k}=40.0$. Durante el primer flujo radial para $\mathrm{q}=90^{\circ}, \mathrm{y}$ usando la Ec. 2.1 resulta $k_{x, 1}=10.47 \mathrm{md}$. Luego, $\mathrm{k}_{\mathrm{x}, 2}=10.47 /$ $*_{0}=0.261 \mathrm{md}$. Del gráfico cartesiano de la Fig. 29, estado pseudoestable, la pendiente es 0.09 ay el corte es 69. De la Ec. 2.10 se tiene $L_{x} L_{y} L_{z}=1.06 \times 10^{8}$ $\mathrm{A}^{2}$. De la Ec. 2.13 resulta una presión promedia $=$ $P_{-}+69 \mathrm{psi}$, y de la $2.14, J=40 / 69=0.58 \mathrm{stb} / \mathrm{d} /$ poi. En la Fig. 2.3, la derivada se desvía a $\mathrm{t}=21 \mathrm{hr}$ Luego, con la Ec. 1.15, la distancia del pozo a la frontera es $481.6 \mathrm{ft}$.

\section{Casa 3: Pozo Dndulante en un Yacimienta Bi-Capas}

La configuración del pozo se da en la Fig. 1. Las principales características son:

1) Durante el primer flujo radial la derivada de presión se expresa mediante dos casos:

Caso A,

(3.1)

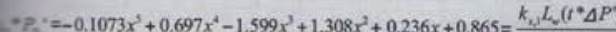

$141.2 q B \mu$

5inde $x=\log \left(k_{x, 1} / k_{x, 2}\right)=\log \left(R_{k}\right.$

Caso B,

$* P_{b}=-0.2184 x^{5}+1.3764 x^{4}-3.097 x^{3}+2.5777 x^{2}+0.2973 x+0.8649$

2. Durante el segundo flujo radial, la magnitud de la derivada, solo para el caso B, se expresa por:

$P_{D}^{\prime}=-0.1455 x^{2}+0.9126 x+1.2667$

3 Durante el periodo de transición antes del estado pseudoestable existe una línea de pendiente o.8. Su longitud es útil para hallar $R_{k}$ mediante:

(3.5)

$=1.451 \times 10^{-1} R_{4}^{3}-3.992 \times 10^{-1} R_{1}^{4}+4.054 \times 10^{-1} R_{4}^{3}-0.00190 R_{1}^{2}+0.0531 R_{1}-0.0512$

- Durante el estado pseudoestable, la presión fímensional está dada por:

$\frac{L_{2}}{L}=2 \pi t_{D A}+I_{p m}$ donde $I_{p s s}$ es una constante que depende de la configuración del pozo, localización del pozo y forma del yacimiento. La anterior ecuación en unidades de campo se convierte en:

$$
\Delta P=\frac{0.234 q B \mu}{\phi \mu_{i} L_{x} L_{y} L_{z}} t+\frac{141.2 q B \mu}{k_{x} L_{z}} I_{s s}
$$

5) La presión promedia durante estado pseudoestable puede expresarse como:

$$
\left(\bar{P}-P_{w f}\right)=\frac{141.2 q B \mu}{k_{x} L_{s}} I_{s s}
$$

6) $J$ se calcula de:

$$
J=\frac{q}{\left(\bar{P}-P_{w f}\right)}=\frac{0.00708 k_{x} L_{x}}{q B \mu I_{p x s}}
$$

El tiempo de desviación de la derivada sirve para estimar la permeabilidad direccional de la Ec. 1.15. El procedimiento paso a paso para hallar los parámetros del yacimiento es:

Paso 1: Haga los mismos gráficos del caso 1. Identifique la transición antes del estado pseudoestable. Determine la longitud de la recta de pendiente 0.8. Halle $R_{k}$ de la Ec. (3.5).

Paso 2: Identifique el primer flujo radial. Use la Ec. (3.1) y $R_{k}$ del paso 1 para hallar las permeabilidades en la direcçión $x$ para ambas capas

Paso 3: Identifique el periodo de estado pseudoestable. En el gráfico cartesiano, Fig. 3.4, trace un recta. Halle su pendiente y corte. Determine el volumen del yacimiento con la Ec. (3.7).

Paso 4: Calcule la presión promedia del yacimiento con la Ec. (3.8).

Paso 5: Use la Ec. (3.9) para determinar $J$.

Paso 6: Calcule la distancia del pozo a la frontera con la Ec. 1.15.

\section{Ejemplo}

Los datos de producción se dan en las Figs. 3.1 y 3.2 para este ejemplo sintético. Los parámetros del pozo, fluido y yacimiento se dan a continuación:

$$
\begin{array}{lll}
L_{w}=272 \mathrm{ft} & q=50 \mathrm{stb} / \mathrm{d} & B=1.2 \mathrm{rb} / \mathrm{stb} \\
\mu=1 \mathrm{cp} & & \\
\phi=0.1 & c_{t}=0.00002 \mathrm{psi}^{-1} & r_{w}-0.5 \mathrm{ft}
\end{array}
$$

Solución: Durante la transición antes del estado pseudoestable existe una línea de pendiente 0.8 cuya 


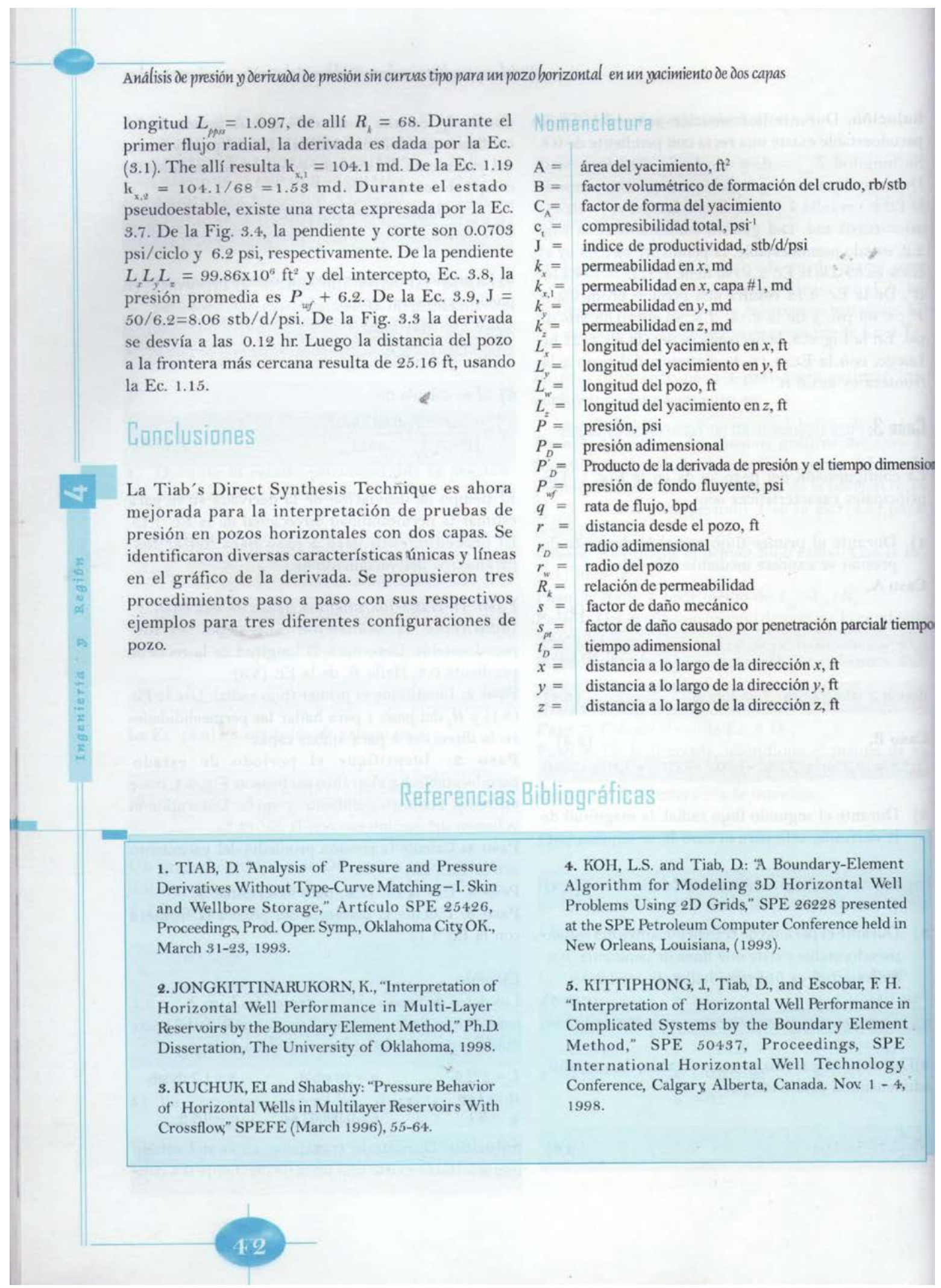


Kittiphong Jongkittinarukorn/Djebbar Tiab/Freddy Humberto Escobar
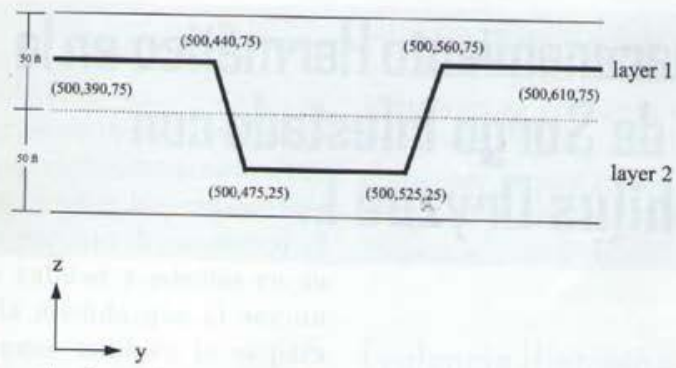

Figura 1. Configuración de pozo horizontal para el caso 3.

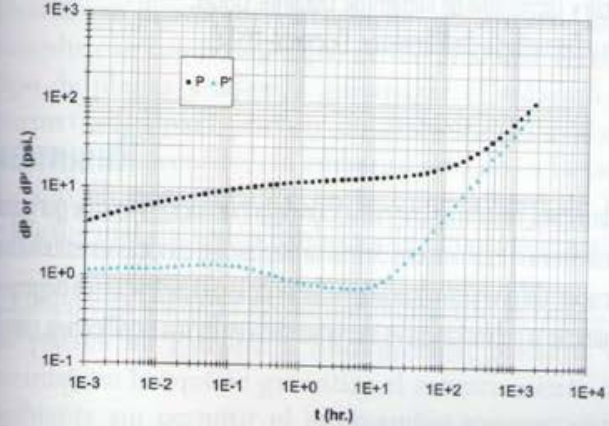

Figura 1.1 Presión y derivada de presión para el caso 1.

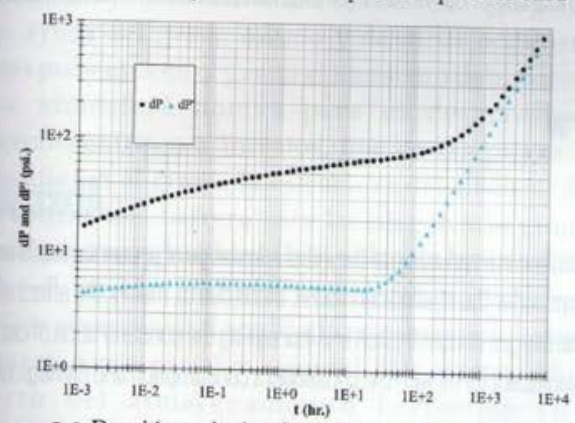

Figura 2.1 Presión y derivada de presión para el caso 1.

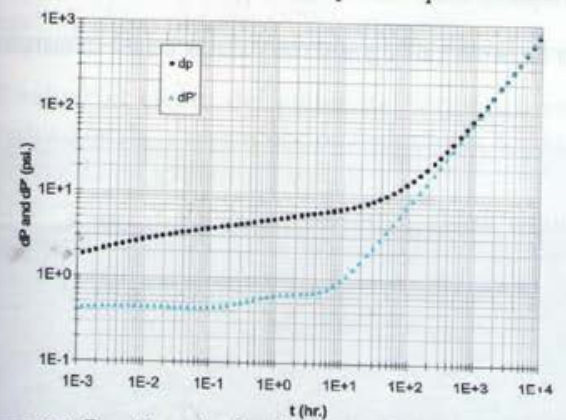

Figura 3.1 Presión y derivada de presión para el caso 3 .

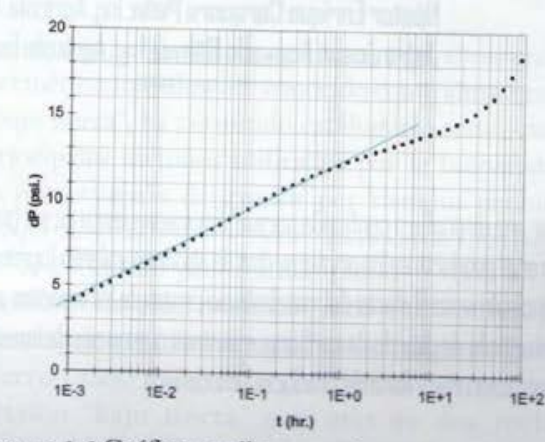

Figura 1.2 Gráfico semilog para el caso 1.

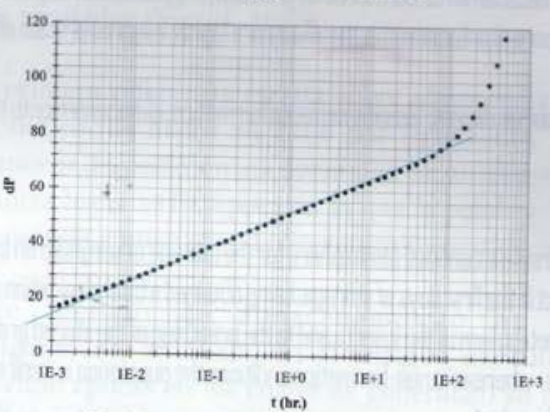

Figura 2.2 Gráfico semilog para el caso 2.

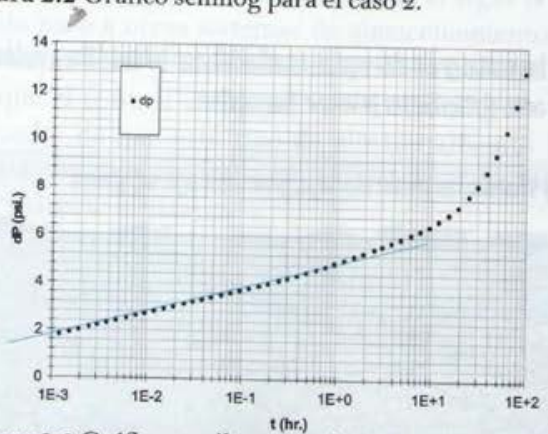

Figura 3.2 Gráfico semilog para el caso 3.

Publicado en: Boletín Estadístico Mensual del ACIPET, No. 6. Junio de 2004-Año 37 -Formato disco compacto 